\title{
Dietary patterns and onset of obesity among medical students in Banja Luka
}

\section{Suzana Savić, ${ }^{1,2}$, Gordana Tešanovićc ${ }^{1,2}$, Larisa Gavran³, Saša Stanivuković ${ }^{4}$}

'Primary Healthcare Center Banja Luka, The Republic of Srpska, Bosnia and Herzegovina

${ }^{2}$ University of Banja Luka, Faculty of Medicine, Department of Family Medicine, The Republic of Srpska, Bosnia and Herzegovina

${ }^{3}$ University of Zenica, Faculty of Medicine, Department of Family Medicine, Bosnia and Herzegovina

${ }^{4}$ University of Banja Luka, Faculty of Medicine, Banja Luka, The Republic of Srpska, Bosnia and Herzegovina

Primljen - Received: 27/03/2020

Prihvaćen - Accepted: 18/11/2020

Corresponding author:

Suzana Savić, MD, PhD

Kordunaška 10, 78000 Banja Luka

suzana.savic@med.unibl.org

Copyright: @2020 Suzana Savić et all. This is an Open Access article distributed under the terms of the Creative Commons Attribution 4.0 International (CC BY 4.0) license.

\begin{abstract}
Introduction. The World Health Organization (WHO) revealed that in 2016 more than 1.9 billion adults, 18 years and older, were overweight. Over 650 million of them were obese. Diet quality in university students is determined by factors such as family, food availability and meals in student canteens. The purpose of this study was to investigate frequency of obesity and its relation to dietary habits, gender and age/year of study among medical students at the Faculty of Medicine of the University of Banja Luka.

Methods. This is a prospective and descriptive study conducted among 1-6 year student population at the Faculty of Medicine of the University of Banja Luka. The study includes a validated survey instrument Youth Risk Behavior Survey Questionnaires. The study was approved by the Ethics Committee of the Faculty of Medicine and carried out as an anonymous survey during the winter term of the academic year 2017/2018.
\end{abstract}

Results. Out of the total 601 students, the research sample included 543 students: 327 female (60.2\%) and 216 (39.8\%) male students. The majority of them (62.1\%) fell within healthy weight range, and $2.4 \%$ were class 1 obesity. Most of female students (75.7\%) had healthy weight while their male counterparts (73.2\%) were overweight. To sum up, $57.5 \%$ of them consumed breakfast every day: female students significantly more (60.6\%) than male students (51.9\%). Third-year students (59.4\%) had breakfast most frequently compared to the fifth-year students (55.0\%) who ate it the least.

Conclusion. The study found a high prevalence of overweight among male respondents compared to their female counterparts, who had predominantly healthy weight.

Keywords: medical student, nutritional status, dietary patterns

\section{Introduction}

Making the right food choices that allow sufficient nutrient intake is a prerequisite for functioning properly throughout study years, particularly due to insufficient physical activity and mental stress [1].

Previous studies have shown that university students do not adhere to dietary guidelines. Modern lifestyle, irregular meals, changes in students' dietary patterns (choice, type, cooking methods and food amounts) in combination with decreased physical activity can increase the risk of developing many potentially serious health conditions, all of which are associated with poor diet [2-4].

Children and adolescents with high body mass index (BMI) are likely to develop cardiovascular diseases later in life $[5,6]$. Obesity and high BMI are associated with the morbidity and mortality of some types of cancer and chronic diseases such as osteoarthritis, liver and kidney disease, sleep apnea, and depression [4,7]. Eating a healthy balanced diet accompanied by regular exercise is essential in the prevention of cardiovascular, malignant diseases and diabetes mellitus [3,8,9]. 
The WHO defines overweight and obesity as abnormal and excessive fat accumulation in the body that may impair health. Obesity is number two cause of death, which can be prevented [10-12].

Weight gain in men is usually associated with fat accumulation in the upper part of the abdomen, chest, neck and jaw, and in women around the stomach, gluteal regions and extremities. The fundamental cause of obesity and overweight is an energy imbalance between calories consumed and calories expended $[5,11,12]$.

The WHO uses BMI index to calculate nutritional status. It is defined as a person's weight in kilograms divided by the square of his height in meters. For adults over 18 years of age, overweight implies a BMI greater than or equal to $25 \mathrm{~kg} / \mathrm{m}^{2}$ [13].

The following factors are associated with obesity: heredity, physical inactivity, an increased intake of energy-dense foods, endocrine and metabolic disorders, traumas, hypothalamic tumors, and iatrogenic disorders caused by regular use of antidepressants and corticosteroids. Other factors that influence obesity are socioeconomic and socio-demographic, as well as dietary behavior [10]. The WHO estimates show that in 2016, more than 1.9 billion adults, 18 years and older, were overweight. Overall, about $13 \%$ of the world's adult population (11\% of men and $15 \%$ of women) were obese in 2016. The worldwide prevalence of obesity nearly tripled between 1975 and 2016 [11,14].

The purpose of this study was to investigate frequency of obesity and its relation to dietary habits, gender and age/year of study among medical students at the Faculty of Medicine of the University of Banja Luka.

\section{Methods}

This is a prospective and descriptive study conducted among the 1-6-year student population of the Faculty of Medicine. The validated survey instrument Youth Risk Behavior Survey Questionnaires (YRBS) was used, including 28 questions that meet study requirements (gender, age, study stage/years) and questions about self-weight assessment and physical activity levels. The paper is one in a series derived from the same study.
The written consent was obtained from the Ethics Committee of the Faculty. The authors conducted the study as an anonymous survey during the winter term of the academic year 2017/2018.

The survey was carried out in accordance with the lectures given by the Vice Dean for Teaching. The questionnaire took $10-20$ minutes to be completed. Out of the total 601 students, 543 of them took part in the survey: Year $1-110$ students, Year 2 - 91 students, Year 3 - 128 students, Year 4 - 98 students, Year 5 - 60 students, Year $6-53$ students.

The data were stored and analyzed using Excel database and SPSS program. Statistical methods included: descriptive usage statistics-frequency, expressed in absolute and percentage numbers, and the differences between individual groups of respondents were tested by the $X^{2}$ test. The statistically significant difference in continuous nonparametric variables between the two groups of subjects was examined using the Mann - Whitney U test. There was a statistical significance between compared data provided that the probability of the null hypothesis was less than $5 \%(p<0.05)$.

\section{Results}

Out of the total 601 students, the study population included 543 students (90.3\%), aged 18-29 years, 327 females $(60.2 \%)$ and $216(39.8 \%)$ males.

BMI by gender and study stage/years profile showed that female students were overweight in the first five study years. Six-year students had healthy weight according to gender profile $(62.5 \%$ females, $16.1 \%$ males). We found difference in study stage/years and gender profile $(\mathrm{p}<0.000)$ (Table 1). 222 (40.9\%) of respondents did not consume fruit juices. 132 female students $(60.3 \%)$ consumed fruit juice once to three times per week and 87 male students (39.7\%), respectively. Fruit juice consumption per week showed no statistical significance where gender profile $(\mathrm{p}=0.355)$ was concerned, while the difference was found in study stage/years profile $(\mathrm{p}=0.000)$.

There was a statistically significant difference in fruit juice consumption where gender profile was concerned $(\mathrm{p}=0.000)$ compared to study stage/years profile $(\mathrm{p}=0.214)$ (Table 2$)$. 
Table 1. BMI classification of respondents by gender and study stage/years

\begin{tabular}{|c|c|c|c|c|c|c|}
\hline \multicolumn{7}{|c|}{ Body mass index - classification } \\
\hline Study stage/years & Gender & Underweight & Normal weight & Overweight & Obesity class 1 & $\mathrm{p}$ \\
\hline \multirow{2}{*}{ Year 1} & M & $1(0.9 \%)$ & $12(10.9 \%)$ & $21(19.1 \%)$ & $2(1.8 \%)$ & \multirow{13}{*}{0.000} \\
\hline & W & $9(8.2 \%)$ & $61(55.5 \%)$ & $4(3.6 \%)$ & $0(0.0 \%)$ & \\
\hline \multirow{2}{*}{ Year 2} & $\mathrm{M}$ & $1(1.1 \%)$ & $11(12.1 \%)$ & $26(28.6 \%)$ & $0(0.0 \%)$ & \\
\hline & W & $5(5.5 \%)$ & $40(44.0 \%)$ & $8(8.8 \%)$ & $0(0.0 \%)$ & \\
\hline \multirow{2}{*}{ Year 3} & M & $0(0.0 \%)$ & $25(19.5 \%)$ & $27(21.1 \%)$ & $4(3.1 \%)$ & \\
\hline & W & $5(3.9 \%)$ & $54(42.2 \%)$ & $12(9.4 \%)$ & $1(0.8 \%)$ & \\
\hline \multirow{2}{*}{ Year 4} & $\mathrm{M}$ & $0(0.0 \%)$ & $14(14.3 \%)$ & $32(32.7 \%)$ & $2(2.0 \%)$ & \\
\hline & W & $2(2.0 \%)$ & $34(34.7 \%)$ & $14(14.3 \%)$ & $0(0.0 \%)$ & \\
\hline \multirow{2}{*}{ Year 5} & $\mathrm{M}$ & $0(0.0 \%)$ & $7(11.7 \%)$ & $9(15.0 \%)$ & $2(3.3 \%)$ & \\
\hline & $\mathrm{W}$ & $3(5.0 \%)$ & $35(58.3 \%)$ & $4(6.7 \%)$ & $0(0.0 \%)$ & \\
\hline \multirow{2}{*}{ Year 6} & $\mathrm{M}$ & $0(0.0 \%)$ & $9(16.1 \%)$ & $5(8.9 \%)$ & $2(3.6 \%)$ & \\
\hline & $\mathrm{W}$ & $3(5.4 \%)$ & $35(62.5 \%)$ & $2(3.6 \%)$ & $0(0.0 \%)$ & \\
\hline Total & & $29(5.4 \%)$ & $337(62.2 \%)$ & $164(30.2 \%)$ & $13(2.4 \%)$ & \\
\hline p (study stage/years & & & 0.002 & & & \\
\hline
\end{tabular}

Table 2. Classification of respondents according to fruit consumption by gender and study stage/years

\begin{tabular}{|c|c|c|c|c|c|c|c|c|c|c|}
\hline \multicolumn{11}{|c|}{ During the past 7 days, how many times did you eat fruit (excluding fruit juice)? } \\
\hline $\begin{array}{l}\text { Study } \\
\text { stage/years }\end{array}$ & Gender & $\begin{array}{l}\text { No } \\
\text { consumption }\end{array}$ & $\begin{array}{l}\text { 1-3 times } \\
\text { a day }\end{array}$ & $\begin{array}{l}\text { 4-6 times } \\
\text { a day }\end{array}$ & $\begin{array}{l}\text { once } \\
\text { a day }\end{array}$ & $\begin{array}{l}2 \text { times } \\
\text { a day }\end{array}$ & $\begin{array}{l}3 \text { times } \\
\text { a day }\end{array}$ & $\begin{array}{l}\geq 4 \text { times } \\
\text { a day }\end{array}$ & Total & $\mathrm{p}$ (gender) \\
\hline \multirow{2}{*}{ Year 1} & M & $\begin{array}{l}6 \\
(5.5 \%) \\
\end{array}$ & $\begin{array}{l}10 \\
(9.1 \%) \\
\end{array}$ & $\begin{array}{l}7 \\
(6.4 \%) \\
\end{array}$ & $\begin{array}{l}9 \\
(8.2 \%) \\
\end{array}$ & $\begin{array}{l}4 \\
(3.6 \%) \\
\end{array}$ & $\begin{array}{l}0 \\
(0.0 \%)\end{array}$ & $\begin{array}{l}1 \\
(0.9 \%)\end{array}$ & $\begin{array}{l}37 \\
(33.6 \%)\end{array}$ & \\
\hline & W & $\begin{array}{l}4 \\
(3.6 \%) \\
\end{array}$ & $\begin{array}{l}19 \\
(17.3 \%) \\
\end{array}$ & $\begin{array}{l}10 \\
(9.1 \%) \\
\end{array}$ & $\begin{array}{l}12 \\
(10.9 \%) \\
\end{array}$ & $\begin{array}{l}12 \\
(10.9 \%) \\
\end{array}$ & $\begin{array}{l}8 \\
(7.3 \%) \\
\end{array}$ & $\begin{array}{l}8 \\
(7.3 \%) \\
\end{array}$ & $\begin{array}{l}73 \\
(66.4 \%) \\
\end{array}$ & \\
\hline \multirow{2}{*}{ Year 2} & M & $\begin{array}{l}5 \\
(5.5 \%) \\
\end{array}$ & $\begin{array}{l}16 \\
(17.6 \%) \\
\end{array}$ & $\begin{array}{l}5 \\
(5.5 \%) \\
\end{array}$ & $\begin{array}{l}5 \\
(5.5 \%) \\
\end{array}$ & $\begin{array}{l}6 \\
(6.6 \%) \\
\end{array}$ & $\begin{array}{l}2 \\
(2.2 \%) \\
\end{array}$ & $\begin{array}{l}1 \\
(1.1 \%) \\
\end{array}$ & $\begin{array}{l}40 \\
(44.0 \%) \\
\end{array}$ & \\
\hline & W & $\begin{array}{l}2 \\
(2.2 \%) \\
\end{array}$ & $\begin{array}{l}6 \\
(6.6 \%) \\
\end{array}$ & $\begin{array}{l}11 \\
(12.1 \%) \\
\end{array}$ & $\begin{array}{l}6 \\
(6.6 \%) \\
\end{array}$ & $\begin{array}{l}12 \\
(13.2 \%) \\
\end{array}$ & $\begin{array}{l}8 \\
(8.8 \%) \\
\end{array}$ & $\begin{array}{l}6 \\
(6.6 \%) \\
\end{array}$ & $\begin{array}{l}51 \\
(56.0 \%) \\
\end{array}$ & \\
\hline \multirow{2}{*}{ Year 3} & M & $\begin{array}{l}8 \\
(6.3 \%) \\
\end{array}$ & $\begin{array}{l}14 \\
(10.9 \%)\end{array}$ & $\begin{array}{l}15 \\
(11.7 \%) \\
\end{array}$ & $\begin{array}{l}10 \\
(7.8 \%)\end{array}$ & $\begin{array}{l}5 \\
(3.9 \%) \\
\end{array}$ & $\begin{array}{l}3 \\
(2.3 \%) \\
\end{array}$ & $\begin{array}{l}1 \\
(0.8 \%) \\
\end{array}$ & $\begin{array}{l}56 \\
(43.8 \%) \\
\end{array}$ & \\
\hline & W & $\begin{array}{l}8 \\
(6.3 \%) \\
\end{array}$ & $\begin{array}{l}14 \\
(10.9 \%)\end{array}$ & $\begin{array}{l}3 \\
(2.3 \%) \\
\end{array}$ & $\begin{array}{l}19 \\
(14.8 \%)\end{array}$ & $\begin{array}{l}14 \\
(10.9 \%)\end{array}$ & $\begin{array}{l}4 \\
(3.1 \%)\end{array}$ & $\begin{array}{l}10 \\
(7.8 \%)\end{array}$ & $\begin{array}{l}72 \\
(56.3 \%)\end{array}$ & \\
\hline \multirow{2}{*}{ Year 4} & M & $\begin{array}{l}12 \\
(12.2 \%)\end{array}$ & $\begin{array}{l}14 \\
(14.3 \%)\end{array}$ & $\begin{array}{l}9 \\
(9.2 \%) \\
\end{array}$ & $\begin{array}{l}7 \\
(7.1 \%) \\
\end{array}$ & $\begin{array}{l}6 \\
(6.1 \%) \\
\end{array}$ & $\begin{array}{l}0 \\
(0.0 \%) \\
\end{array}$ & $\begin{array}{l}0 \\
(0.0 \%) \\
\end{array}$ & $\begin{array}{l}48 \\
(49.0 \%)\end{array}$ & 0.000 \\
\hline & W & $\begin{array}{l}0 \\
(0.0 \%) \\
\end{array}$ & $\begin{array}{l}7 \\
(7.1 \%) \\
\end{array}$ & $\begin{array}{l}12 \\
(12.4 \%)\end{array}$ & $\begin{array}{l}7 \\
(7.1 \%) \\
\end{array}$ & $\begin{array}{l}10 \\
(10.2 \%)\end{array}$ & $\begin{array}{l}7 \\
(7.1 \%) \\
\end{array}$ & $\begin{array}{l}7 \\
(7.1 \%) \\
\end{array}$ & $\begin{array}{l}50 \\
(51.0 \%)\end{array}$ & \\
\hline \multirow{2}{*}{ Year 5} & M & $\begin{array}{l}3 \\
(5.0 \%) \\
\end{array}$ & $\begin{array}{l}9 \\
(15.0 \%)\end{array}$ & $\begin{array}{l}3 \\
(5.0 \%) \\
\end{array}$ & $\begin{array}{l}2 \\
(3.3 \%)\end{array}$ & $\begin{array}{l}1 \\
(1.7 \%) \\
\end{array}$ & $\begin{array}{l}0 \\
(0 \%) \\
\end{array}$ & $\begin{array}{l}0 \\
(0 \%)\end{array}$ & $\begin{array}{l}18 \\
(30.0 \%)\end{array}$ & \\
\hline & W & $\begin{array}{l}6 \\
(10.0 \%)\end{array}$ & $\begin{array}{l}10 \\
(16.7 \%)\end{array}$ & $\begin{array}{l}4 \\
(6.7 \%) \\
\end{array}$ & $\begin{array}{l}10 \\
(16.7 \%)\end{array}$ & $\begin{array}{l}6 \\
(10.0 \%)\end{array}$ & $\begin{array}{l}4 \\
(6.7 \%) \\
\end{array}$ & $\begin{array}{l}2 \\
(3.3 \%) \\
\end{array}$ & $\begin{array}{l}42 \\
(70.0 \%)\end{array}$ & \\
\hline \multirow{2}{*}{ Year 6} & M & $\begin{array}{l}0 \\
(0.0 \%) \\
\end{array}$ & $\begin{array}{l}2 \\
(3.6 \%) \\
\end{array}$ & $\begin{array}{l}3 \\
(5.4 \%) \\
\end{array}$ & $\begin{array}{l}7 \\
(12.5 \%) \\
\end{array}$ & $\begin{array}{l}3 \\
(5.4 \%) \\
\end{array}$ & $\begin{array}{l}1 \\
(1.8 \%) \\
\end{array}$ & $\begin{array}{l}1 \\
(1.8 \%) \\
\end{array}$ & $\begin{array}{l}17 \\
(30.4 \%)\end{array}$ & \\
\hline & W & $\begin{array}{l}1 \\
(1.8 \%) \\
\end{array}$ & $\begin{array}{l}5 \\
(8.9 \%) \\
\end{array}$ & $\begin{array}{l}7 \\
(12.5 \%) \\
\end{array}$ & $\begin{array}{l}13 \\
(23.2 \%) \\
\end{array}$ & $\begin{array}{l}10 \\
(17.9 \%) \\
\end{array}$ & $\begin{array}{l}1 \\
(1.8 \%) \\
\end{array}$ & $\begin{array}{l}2 \\
(3.6 \%) \\
\end{array}$ & $\begin{array}{l}39 \\
(69.6 \%) \\
\end{array}$ & \\
\hline Total & & $\begin{array}{l}55 \\
(10.1 \%)\end{array}$ & $\begin{array}{l}126 \\
(23.2 \%)\end{array}$ & $\begin{array}{l}88 \\
(16.2 \%)\end{array}$ & $\begin{array}{l}107 \\
(19.7 \%)\end{array}$ & $\begin{array}{l}90 \\
(16.6 \%)\end{array}$ & $\begin{array}{l}38 \\
(7.0 \%)\end{array}$ & $\begin{array}{l}39 \\
(7.2 \%)\end{array}$ & $\begin{array}{l}543 \\
(100 \%)\end{array}$ & \\
\hline $\begin{array}{l}\mathrm{p} \text { (study } \\
\text { stage/years) }\end{array}$ & \multicolumn{10}{|c|}{0.214} \\
\hline
\end{tabular}


Table 3. Classification of respondents according to milk consumption by gender and study stage/years

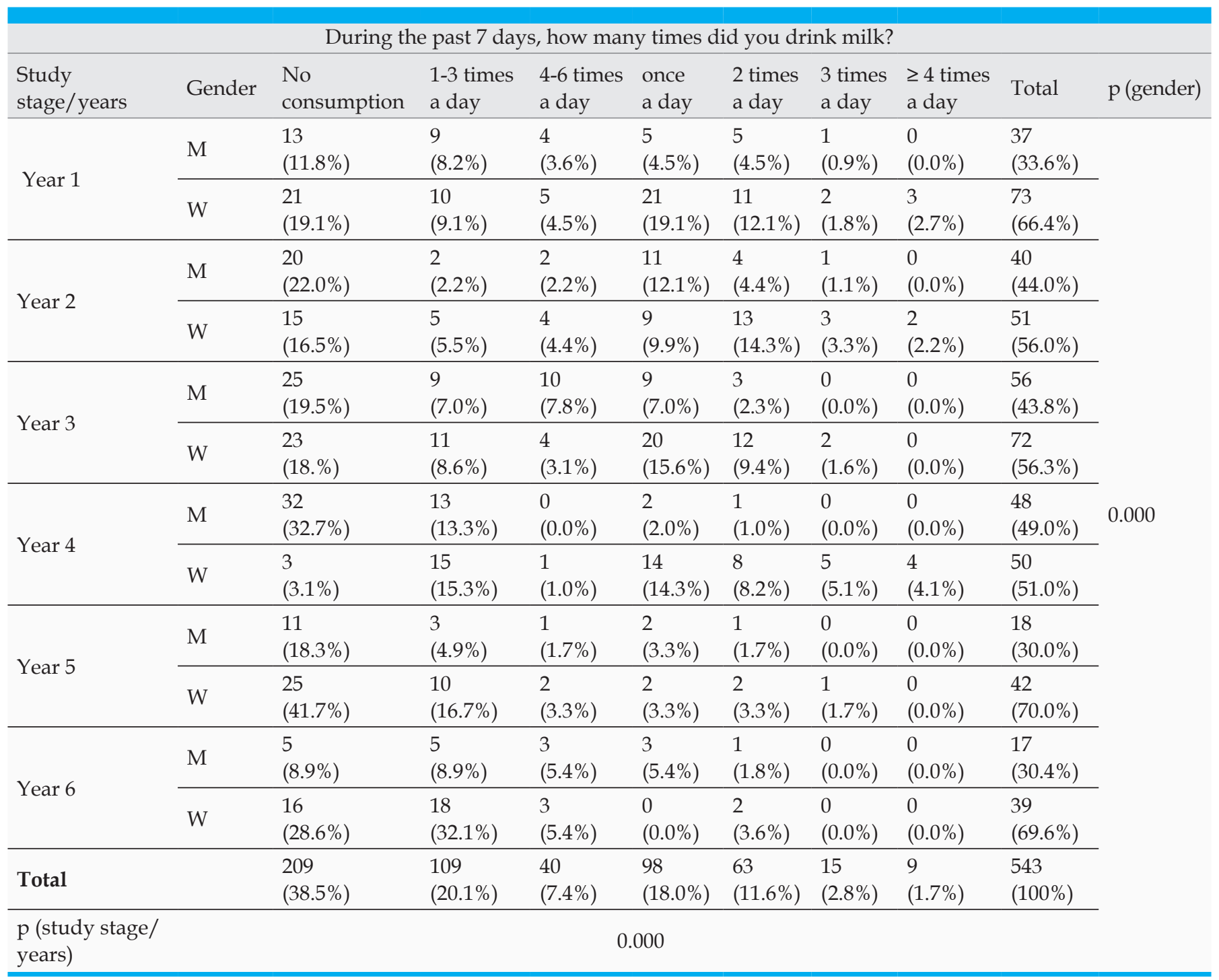

Most of the respondents, $279(51.4 \%)$, did not consume lettuce. 108 females $(60.3 \%)$ consumed lettuce once to three times per week and 71 males $(39.7 \%)$, respectively. Lettuce consumption per week showed statistical significance where gender $(p=0.002)$ and study stage/years profile were concerned $(\mathrm{p}=0.009)$.

We found difference in weekly consumption of potato in gender and study stage/years profile $(p=0.000)$ while $52.3 \%$ of male and female students reported they did not consume carrot. Most of male and female students (33.3\%) reported they consumed carrot once to three times per week. We found difference in gender $(p=0.020)$ and study stage/years profile $(\mathrm{p}=0.001)$. 1-5-year students (36.5\%) reported they consumed 'other types of vegetable' once to three times per week, while six-year students, 6 of them $(10.7 \%)$, reported they consumed 'other types of vegetable' four to six times per week. We found difference in gender $(p=0.004)$ and study stage/ years profile $(p=0.000)$.

Soda consumption per week demonstrated difference in gender $(p=0.000)$ and study stage/ years profile $(\mathrm{p}=0.003)$.

The percentage of males and females who did not consume milk throughout the week was $38.5 \%$. We found difference in gender $(\mathrm{p}=0.000)$ and study stage/years profile $(\mathrm{p}=0.000)$ (Table 3$)$.

1-6-year students $(57.1 \%)$ reported they con- 
Table 4. Classification of respondents according to breakfast patterns by gender and study stage/years

\begin{tabular}{|c|c|c|c|c|c|c|c|c|c|c|c|}
\hline \multicolumn{12}{|c|}{ During the past 7 days, how many times did you have breakfast? } \\
\hline $\begin{array}{l}\text { Study stage/ } \\
\text { years }\end{array}$ & Gender & 0 days & 1 day & 2 days & 3 days & 4 days & 5 days & 6 days & 7 days & Total & $\mathrm{p}$ (gender) \\
\hline \multirow{2}{*}{ Year 1} & $\mathrm{M}$ & $\begin{array}{l}3 \\
(2.7 \%)\end{array}$ & $\begin{array}{l}0 \\
(0.0 \%)\end{array}$ & $\begin{array}{l}1 \\
(0.9 \%)\end{array}$ & $\begin{array}{l}1 \\
(0.9 \%)\end{array}$ & $\begin{array}{l}1 \\
(0.9 \%)\end{array}$ & $\begin{array}{l}5 \\
(4.5 \%) \\
\end{array}$ & $\begin{array}{l}1 \\
(0.9 \%)\end{array}$ & $\begin{array}{l}25 \\
(22.7 \%)\end{array}$ & $\begin{array}{l}37 \\
(33.6 \%) \\
\end{array}$ & \\
\hline & W & $\begin{array}{l}5 \\
(4.5 \%)\end{array}$ & $\begin{array}{l}0 \\
(0.0 \%)\end{array}$ & $\begin{array}{l}4 \\
(3.6 \%)\end{array}$ & $\begin{array}{l}2 \\
(1.8 \%)\end{array}$ & $\begin{array}{l}5 \\
(4.5 \%)\end{array}$ & $\begin{array}{l}9 \\
(8.2 \%)\end{array}$ & $\begin{array}{l}7 \\
(6.4 \%)\end{array}$ & $\begin{array}{l}41 \\
(37.3 \%)\end{array}$ & $\begin{array}{l}73 \\
(66.4 \%)\end{array}$ & \\
\hline \multirow{2}{*}{ Year 2} & $\mathrm{M}$ & $\begin{array}{l}3 \\
(3.3 \%)\end{array}$ & $\begin{array}{l}0 \\
(0.0 \%)\end{array}$ & $\begin{array}{l}0 \\
(0.0 \%)\end{array}$ & $\begin{array}{l}4 \\
(4.4 \%)\end{array}$ & $\begin{array}{l}6 \\
(6.6 \%)\end{array}$ & $\begin{array}{l}5 \\
(5.5 \%)\end{array}$ & $\begin{array}{l}1 \\
(1.1 \%)\end{array}$ & $\begin{array}{l}21 \\
(23.1 \%)\end{array}$ & $\begin{array}{l}40 \\
(44.0 \%)\end{array}$ & \\
\hline & W & $\begin{array}{l}3 \\
(3.3 \%) \\
\end{array}$ & $\begin{array}{l}0 \\
(0.0 \%)\end{array}$ & $\begin{array}{l}1 \\
(1.1 \%) \\
\end{array}$ & $\begin{array}{l}2 \\
(2.2 \%) \\
\end{array}$ & $\begin{array}{l}6 \\
(6.6 \%)\end{array}$ & $\begin{array}{l}3 \\
(3.3 \%) \\
\end{array}$ & $\begin{array}{l}4 \\
(4.4 \%) \\
\end{array}$ & $\begin{array}{l}32 \\
(35.2 \%) \\
\end{array}$ & $\begin{array}{l}51 \\
(56.0 \%) \\
\end{array}$ & \\
\hline \multirow{2}{*}{ Year 3} & $\mathrm{M}$ & $\begin{array}{l}2 \\
(1.6 \%) \\
\end{array}$ & $\begin{array}{l}1 \\
(0.8 \%)\end{array}$ & $\begin{array}{l}1 \\
(0.8 \%) \\
\end{array}$ & $\begin{array}{l}2 \\
(1.6 \%) \\
\end{array}$ & $\begin{array}{l}3 \\
(2.3 \%) \\
\end{array}$ & $\begin{array}{l}11 \\
(8.6 \%) \\
\end{array}$ & $\begin{array}{l}5 \\
(3.9 \%) \\
\end{array}$ & $\begin{array}{l}31 \\
(24.2 \%) \\
\end{array}$ & $\begin{array}{l}56 \\
(43.8 \%) \\
\end{array}$ & \\
\hline & W & $\begin{array}{l}7 \\
(5.5 \%)\end{array}$ & $\begin{array}{l}0 \\
(0.0 \%)\end{array}$ & $\begin{array}{l}2 \\
(1.6 \%)\end{array}$ & $\begin{array}{l}2 \\
(1.6 \%)\end{array}$ & $\begin{array}{l}6 \\
(4.7 \%)\end{array}$ & $\begin{array}{l}6 \\
(4.7 \%) \\
\end{array}$ & $\begin{array}{l}4 \\
(3.1 \%)\end{array}$ & $\begin{array}{l}45 \\
(35.2 \%)\end{array}$ & $\begin{array}{l}72 \\
(56.3 \%)\end{array}$ & \\
\hline \multirow{2}{*}{ Year 4} & $\mathrm{M}$ & $\begin{array}{l}5 \\
(5.1 \%) \\
\end{array}$ & $\begin{array}{l}1 \\
(1.0 \%) \\
\end{array}$ & $\begin{array}{l}3 \\
(3.1 \%) \\
\end{array}$ & $\begin{array}{l}6 \\
(6.1 \%) \\
\end{array}$ & $\begin{array}{l}7 \\
(7.1 \%) \\
\end{array}$ & $\begin{array}{l}8 \\
(8.2 \%) \\
\end{array}$ & $\begin{array}{l}4 \\
(4.1 \%) \\
\end{array}$ & $\begin{array}{l}14 \\
(14.3 \%)\end{array}$ & $\begin{array}{l}48 \\
(49.0 \%) \\
\end{array}$ & 0.190 \\
\hline & W & $\begin{array}{l}2 \\
(2.0 \%)\end{array}$ & $\begin{array}{l}2 \\
(2.0 \%)\end{array}$ & $\begin{array}{l}1 \\
(1.0 \%)\end{array}$ & $\begin{array}{l}1 \\
(1.0 \%)\end{array}$ & $\begin{array}{l}6 \\
(6.1 \%)\end{array}$ & $\begin{array}{l}4 \\
(4.1 \%)\end{array}$ & $\begin{array}{l}6 \\
(6.1 \%)\end{array}$ & $\begin{array}{l}28 \\
(28.6 \%)\end{array}$ & $\begin{array}{l}50 \\
(51.0 \%)\end{array}$ & \\
\hline \multirow{2}{*}{ Year 5} & $\mathrm{M}$ & $\begin{array}{l}0 \\
(0.0 \%) \\
\end{array}$ & $\begin{array}{l}1 \\
(1.7 \%) \\
\end{array}$ & $\begin{array}{l}1 \\
(1.7 \%) \\
\end{array}$ & $\begin{array}{l}2 \\
(3.3 \%) \\
\end{array}$ & $\begin{array}{l}2 \\
(3.3 \%) \\
\end{array}$ & $\begin{array}{l}2 \\
(3.3 \%) \\
\end{array}$ & $\begin{array}{l}2 \\
(3.3 \%) \\
\end{array}$ & $\begin{array}{l}8 \\
(13.3 \%) \\
\end{array}$ & $\begin{array}{l}18 \\
(30.0 \%) \\
\end{array}$ & \\
\hline & W & $\begin{array}{l}0 \\
(0.0 \%) \\
\end{array}$ & $\begin{array}{l}0 \\
(0.0 \%)\end{array}$ & $\begin{array}{l}3 \\
(5.0 \%) \\
\end{array}$ & $\begin{array}{l}7 \\
(11.7 \%) \\
\end{array}$ & $\begin{array}{l}2 \\
(3.3 \%) \\
\end{array}$ & $\begin{array}{l}2 \\
(3.3 \%) \\
\end{array}$ & $\begin{array}{l}3 \\
(5.0 \%) \\
\end{array}$ & $\begin{array}{l}25 \\
(41.7 \%)\end{array}$ & $\begin{array}{l}42 \\
(70.0 \%)\end{array}$ & \\
\hline \multirow{2}{*}{ Year 6} & $\mathrm{M}$ & $\begin{array}{l}0 \\
(0 \%) \\
\end{array}$ & $\begin{array}{l}0 \\
(0.0 \%) \\
\end{array}$ & $\begin{array}{l}1 \\
(1.8 \%) \\
\end{array}$ & $\begin{array}{l}1 \\
(1.8 \%) \\
\end{array}$ & $\begin{array}{l}0 \\
(0.0 \%) \\
\end{array}$ & $\begin{array}{l}0 \\
(0.0 \%) \\
\end{array}$ & $\begin{array}{l}2 \\
(3.6 \%) \\
\end{array}$ & $\begin{array}{l}13 \\
(23.2 \%) \\
\end{array}$ & $\begin{array}{l}17 \\
(30.4 \%) \\
\end{array}$ & \\
\hline & W & $\begin{array}{l}1 \\
(1.8 \%) \\
\end{array}$ & $\begin{array}{l}0 \\
(0.0 \%)\end{array}$ & $\begin{array}{l}2 \\
(3.6 \%) \\
\end{array}$ & $\begin{array}{l}1 \\
(1.8 \%) \\
\end{array}$ & $\begin{array}{l}0 \\
(0.0 \%) \\
\end{array}$ & $\begin{array}{l}3 \\
(5.4 \%) \\
\end{array}$ & $\begin{array}{l}5 \\
(8.9 \%) \\
\end{array}$ & $\begin{array}{l}27 \\
(48.2 \%) \\
\end{array}$ & $\begin{array}{l}39 \\
(69.6 \%) \\
\end{array}$ & \\
\hline Total & & $\begin{array}{l}31 \\
(5.7 \%) \\
\end{array}$ & $\begin{array}{l}5 \\
(0.9 \%) \\
\end{array}$ & $\begin{array}{l}20 \\
(3.7 \%) \\
\end{array}$ & $\begin{array}{l}31 \\
(5.7 \%) \\
\end{array}$ & $\begin{array}{l}44 \\
(8.1 \%)\end{array}$ & $\begin{array}{l}58 \\
(10.7 \%) \\
\end{array}$ & $\begin{array}{l}44 \\
(8.1 \%) \\
\end{array}$ & $\begin{array}{l}310 \\
(57.1 \%) \\
\end{array}$ & $\begin{array}{l}543 \\
(100 \%) \\
\end{array}$ & \\
\hline $\begin{array}{l}\mathrm{p} \text { (study } \\
\text { stage/years) }\end{array}$ & & & & & & 012 & & & & & \\
\hline
\end{tabular}

sumed breakfast every day. There was no statistical significance where gender profile was concerned ( $p=0.190)$, but a statistically significant difference was found in study stage/years $(\mathrm{p}=0.012)($ Table 4$)$.

\section{Discussion}

During the study, each student spends more time sitting, compared to average citizen. Also, taking the exams causes certain level of anxiety. These factors can induce obesity especially when they are present during long period of time. Academic studying change eating habits compared to previous period (many students move to another city - separation from family members).
The sample consisted of 543 students. 327 of them (60.2\%) were female and $216(39.8 \%)$ male students. The majority of them $(62.1 \%)$ had healthy weight, $30.2 \%$ were overweight, $5.4 \%$ underweight and $2.4 \%$ belonged to class 1 obesity. Most of females (75.7\%) had normal weight, while their male counterparts (73.2\%) were overweight. Similar findings were reported by Prišlin et al. in a study conducted at the Faculty of Veterinary Medicine in Zagreb among second-year students over the course of three academic years 2014-2017. The study found the prevalence of normal weight $81.7 \%$, overweight $8.06 \%$, class 1 obesity $1.09 \%$, and underweight $9.16 \%$ [1]. In another study, conducted on a sample of 248 students from Rijeka in 2011-12, Čulin and Anđelić-Breš found the 
prevalence of normal weight $70.6 \%$, overweight $17.6 \%$, class 1 obesity $10.2 \%$ and underweight $1.6 \%$. Similar results were reported across gender match, normal weight $75.6 \%$ (females) and class 1 obesity $17.9 \%$ (males) [15]. Crnobrnja et al. carried out a study on students from the University of Novi Sad in 2012 and found the prevalence of normal weight $73.3 \%$, overweight $22 \%$, class 1 obesity $1.08 \%$, and underweight $4.58 \%$. The prevalence of overweight $(41.7 \%)$ and obesity $(1.9 \%)$ was higher among male compared to their female counterparts, which corresponds to our findings [16]. Likewise et al. conduced a study on students from Novi Sad and found the prevalence of overweight and obesity to be higher among male than in female students (overweight $33.5 \%$, obesity $7.5 \%$ ) [17]. The findings by Grujić et al, a study that included the population from Vojvodina, aged 20-29 years old, showed that overweight among males was $29.6 \%$ and $11.5 \%$ among females, obesity was $9.7 \%$ and $7.7 \%$, respectively [18]. The study from Niš showed a high prevalence of overweight among male students $(38.2 \%)$ and $7.95 \%$ among female students, while obesity was $7.2 \%$ and $1.32 \%$, respectively [19].

Our results demonstrate that $40.9 \%$ of respondents did not consume fruit juices $(60.3 \%$ females, $39.7 \%$ males) 30 days prior to the survey. Out of them, the highest percentage belonged to third-year students ( $25.8 \%$ females, $21.1 \%$ males). $49.2 \%$ of respondents did not consume soda (35.2\% males, $58.4 \%$ females). The percentage of students who consumed soda once to three times per week was $30.0 \%$. There were $23.2 \%$ of respondents who consumed fruit once to three times per week. $7.2 \%$ respondents consumed fruit more than four times per week. The percentage of students who consumed vegetables once to three times per week (lettuce, potato, carrot) was $36.6 \%: 34.2 \%$ males and $39.4 \%$ females, respectively, predominantly third-year students $(40.7 \%)$. More than a half of them $(51.4 \%)$ reported they consumed lettuce while $52.3 \%$ ate carrot, predominantly fifth-year students. Respondents consumed lettuce and carrot once to three times per week. "Other types of vegetable" were consumed by $36.5 \%$ of respondents once to three times a week. Out of them $42.6 \%$ were male students and $32.4 \%$ female students, predominantly third-year students (35.9\%). Sim- ilar findings were demonstrated where milk consumption was concerned. $38.5 \%$ of respondents did not consume milk, predominantly men and third-year students (37.5\%). There were 20.1\% of students who consumed milk once to three times per week, predominantly women and fourth-year students (28.6\%). Breakfast patterns showed that $5.7 \%$ did not have breakfast at all (6\% males, $5.5 \%$ females). Nevertheless, most of them (57.5\%) had breakfast every day: $60.6 \%$ of them were females students and $51.9 \%$ were males. The percentage of respondents who consumed breakfast was highest among third-year students $(59.4 \%)$, while the lowest one was recorded among fifth-year students (55.0\%). A study conducted in Kansas by Terry et al, in which YRBS Questionnaires were used, demonstrated that $69.4 \%$ respondents consumed less than five portions of fruit and vegetable every day $(69.4 \%$ males, $69.5 \%$ females) [20]. Likewise et al. surveyed students from College of Medicinal Professional Studies in Belgrade and found that two thirds consumed vegetable every day, while fruit was consumed by more than one third. A total of $17.5 \%$ consumed fruit juices and soda every day, while $31.2 \%$ did not. Less than a half consumed milk every day, and more than a half consumed breakfast every day [21]. Prišlin et al. found that $60.1 \%$ of respondents consumed vegetable every day, males $61.4 \%$, females $59.7 \%$. A total of $25.7 \%$ consumed milk every day (20.9\% males, $26 \%$ females), while $52.6 \%$ consumed breakfast every day (45.6\% males, $54.4 \%$ females) [1]. Banožić et al. surveyed students from the University of Mostar and found that the percentage of those who consumed fruit two to four times per week was $35.7 \%$ while $33.8 \%$ of respondents consumed vegetable six to seven times per week, predominantly women (31.9\%). Fruit juice consumption was more prevalent among the female population $(9.9 \%)$, compared to male students $(6.4 \%)$ [2].

In another study conducted by Kukić et al. in the Municipality of Travnik, the results showed that $25.5 \%$ of females consumed breakfast regularly and $21.1 \%$ of males, respectively. The percentage of females who consumed fruit every day was $49.6 \%$ and $38.9 \%$ for males, respectively. Vegetable consumption accounted for $34.5 \%$ of females and 32.2\% males, respectively. As high 
as $34.5 \%$ of females consumed milk every day and $36.7 \%$ of males, respectively. The proportion of females who consumed juice was $14.4 \%$ and $20 \%$ of males, respectively [3].

Gavran et al. surveyed a sample of 199 students from Zenica and reported that $27.7 \%$ of adolescents consumed fruit rarely or never, $53.3 \%$ consumed vegetable irregularly, $84.4 \%$ consumed breakfast very day, and $64.8 \%$ consumed milk rarely or never [22].

\section{Conclusions}

Breakfast is consumed every day by a larger number of female students compared to males, thirdyear students consumed breakfast the most, while

Funding source. The authors received no specific funding for this work.

Ethical approval. The Ethics Committee of the Faculty of Medicine at the University of Banja Luka approved the study and informed consent was obtained from all

\section{References}

1. Prišlin M, Pincan L, Šiftar O, Vugrovečki SA, Radin L, Vranković L, Aladrović J. Životne, prehrambene navike i stavovi studenata druge godine studija veterinarske medicine. Veterinar 2017;2(55):21-30.

2. Banožić M, Ljubić A, Pehar M, Ištuk J, Kenjerić ČD Prehrambene navike studenata sveučilišta u Mostaru. Hrana u zdravlju i bolesti - stručni časopis za nutricionizam i dijetetiku 2015;4(2):105-12.

3. Kukić E, Karakaš S, Paklarčić M. Razlike u prehrambenim navikama kod učenika uzrasta 15-18 godina u odnosu na spol na prostoru općine Travnik. Hrana u zdravlju i bolesti - stručni časopis za nutricionizam i dijetetiku 2016;5(1):6-14.

4. Šabanović M, Beganlić A, Mulavdić N, Đaković M. Uticaj načina prehrane i fizičke aktivnosti na indeks tjelasne mase $u$ adolescenta. Hrana u zdravlju i bolesti - stručni časopis za nutricionizam i dijetetiku 2016;1(1):10-21.

5. Baker JL, Olsen LW, Sorensen TI. Childhood body mass index and the risk of coronary heart disease in adulthood. N Engl J Med 2012;357:2329-37.

6. Bjorge T, Engeland A, Tverdal A, Davey Smith G. Body mass index in adolescence in relation to cause - specific mortality: a follow-up of 230000 Norwegian adolescents. Am J Epidemiol 2013;168:30-7. fifth-year students consume it the least. Most of the respondents did not consume lettuce, and less than a half of the respondents did not consume fruit juices. Less than a half of male and female students did not consume milk during the week. The study found a high prevalence of overweight among male respondents compared to their female counterparts, who had predominantly healthy weight. This study (one in a series derived from the same research) should lead to better understanding of the onset of obesity due to poor diet among the students of the Faculty of Medicine in Banja Luka and promote preventive measures that would improve the health of this population group.

the individual respondents. The research was conducted according to the Declaration of Helsinki.

Conflicts of interest. The authors declare no conflict of interest.

7. Owen CG, Whincup PH, Ofrei L, Chou QA, Rudnicka AR, Wathern AK et al. Is bodymass index before middle age related to coronary heart disease risk in later life? Evidence from observational studies. Int J Obes 2014;33:866-77.

8. Pi - Sunyer $X$. The medical risk of obesity. Postgard Med 2014;121(6):21-33.

9. Đurić D, Mirković M, Ilić A, Ilić D, Ćorac A, Milošević J. Navike u ishrani i fizičkoj aktivnosti studentske populacije. Praxix Medica 2013;42:33-39.

10. Halilović J, Halilović S, Begić A, Hadžiefendić V. Stepen uhranjenosti odraslog stanovništva Tuzle. Naučna revija, Revija za zdravstvene i tehničke nauke 2016;2(1):23-31.

11. World Health Organization. Obesity and overweight. Geneva: WHO; 2018.

12. Tkalec J, Mikulić Golek M. Prekomjerna tjelasna težina i pretilost djece. U: XXII Kongres obiteljske medicine sa međunarodnim sudjelovanjem: Metabolički sindrom, polipragmazija. Varaždin; 2015. p. 240-48.

13. Stanetić K. Gojaznost. U: Stanetić K, ur. Prevencija u radu porodičnog ljekara. Banja Luka: Univerzitet $\mathrm{u}$ Banjoj Luci, Medicinski fakultet, Narodna i univerzitetska biblioteka Republike Srpske; 2015. p. 40-54. 
14. Lolić A, Tešanović $G$, Vulić D, Stojisavljević D, Štrkić D, Štrbac S, Popović Subotić A, Šeranić A. Gojaznost kod odraslih. Banja Luka: Ministarstvo zdravlja i socijalne zaštite Republike Srpske; 2015.

15. Čulina T, Anđelić Breš S. Povezanost samopoštovanja sa prehrambenim navikama, uhranjenošću, sportom, spolom i dobi riječkih adolescenata. Med Jad 2014;44(1-2):5-12.

16. Crnobrnja V, Sredić B, Dujaković F, Andrejević B. Analiza učestalosti rizičnih oblika gojaznosti kod studenata novosadskog univerziteta. Med Pregl 2012;65(3-4):133-137.

17. Simić $\mathrm{S}$, Vasić $\mathrm{G}$, Jakonić D. Telesna visina, telesna masa i uhranjenost studenata Univerziteta u Novom Sadu. Med danas 2012;9(4-6):141.

18. Grujić V, Martinov Cvejin M, Ač Nikolić E. Udru- ženost gojaznosti sa socioekonomskim faktorima i stilovima života. Vojnosanit Pregl 2012;66(9):705-10.

19. Stojanović T, Višnjić A, Mitrović V, Stojanović M. Faktori rizika za nastanak oboljenja kardiovaskularnog sistema u studentskoj populaciji. Vojnosanit Pregl 2012;66(6):453-58.

20. Huang T, Harris K, Lee R, Nazir N, Born W, Kaur $\mathrm{H}$. Assessing overweight, obesity, diet and physical activity in collage students. J Am Coll Health 2012;52(2):83-86.

21. Dimoski Z, Majstorović B, Kocić S, Radovanović S. Nutritivne navike studenata. Materija Medica 2013;27(2):310-17.

22. Gavran L, Ćerim V, Hasanica N. Can a place of living of elementary school students determine their health habit? Med Glas 2019;16(1):137-43.

\title{
Prehrambene navike i početak gojaznosti među studentima medicine u Banjoj Luci
}

\author{
Suzana Savić1,2, Gordana Tešanović1,2, Larisa Gavran³, Saša Stanivuković ${ }^{4}$ \\ 'Dom zdravlja Banja Luka, Republika Srpska, Bosna i Hercegovina \\ ${ }^{2}$ Univerzitet u Banjoj Luci, Medicinski fakultet, Odsjek Porodična medicina, Republika Srpska, \\ Bosna i Hercegovina \\ ${ }^{3}$ Univerzitet u Zenici, Medicinski fakultet, Odsjek Porodična medicina, Bosna i Hercegovina \\ ${ }^{4}$ Univerzitet u Banjoj Luci, Medicinski fakultet, Banja Luka, Republika Srpska, Bosna i Hercegovina
}

Uvod. Prema podacima Svjetske zdravstvene organizacije iz 2016. godine, više od 1.9 milijardi odraslih osoba iznad 18 godina ima prekomjernu tjelesnu težinu, više od 650 miliona su gojazni. Kvalitet ishrane tokom studiranja prvenstveno zavisi od navika stečenih u porodici, dostupnosti namirnica i obroka koje konzumiraju u restoranima studentske ishrane. Cilj studije je bio da se ispita prisustvo gojaznosti i primjena pravilne ishrane kod studenata Medicinskog fakulteta Univerziteta u Banjoj Luci u odnosu na pol i godine studija.

Metode. Istraživanje je prospektivno desktriptivna studija provedena na studentskoj populaciji svih šest godina studijskog programa medicina Medicinskog fakulteta Univerziteta u Banjoj Luci upotrebom validiranog upitnika - Upitnik za istraživanje rizika ponašanja mladih (Youth Risk Behavior Survey Questionnaires). Studija se provodila nakon odobrenja Etičkog komiteta medicinskog fakulteta, po tipu anonimnog anketiranja, tokom zimskog semestra školske 2017/2018. godine.

Rezultati. Od ukupnog broja studenata (601) u istraživanju je učestvovalo 543 studenta, 327 (60,2\%) ženskog i 216 (39,8\%) muškog pola. Najveći procenat ispitanika $(62,1 \%)$ je bio normalno uhranjen, a 2,4\% ispitanika je imalo gojaznost I stepena. Ženski ispitanici su u najvećem procentu $(75,7 \%)$ bili normalno uhranjeni, a muški (73,2\%) su imali prekomjernu tjelesnu težinu. Najveći dio ispitanika, $57,5 \%$, svakodnevno je doručkovalo, više ispitanici ženskog pola $(60,6 \%)$ u odnosu na ispitanike muškog pola $(51,9 \%)$. $U$ odnosu na godine studije, svakodnevno doručkuju u najvećem procentu studenti treće godine $(59,4 \%)$, a u najmanjem procentu studenti pete godine $(55,0 \%)$.

Zaključak. Prekomjerna tjelesna težina je bila prisutnija kod ispitanika muškog pola, dok su ispitanici ženskog pola u najvećem procentu bili normalno uhranjeni.

Ključne riječi: student medicine, stepen uhranjenosti, navike u ishrani 